Ann. Sci. for., 1983, 40 (4), 325-336

\title{
Influence d'un dessèchement du sol sur la nutrition minérale de jeunes plants de résineux
}

\author{
M. BECKER et G. LEVY * \\ avec la collaboration technique de C. BRECHET **, Renée-France CLAUDE * \\ et Y. LEFËVRE ** \\ I.N.R.A, Laboratoire de Phytoécologie forestière \\ * Station de Recherches sur les sols forestiers et la Fertilisation \\ Centre de Recherches forestières de Nancy \\ Champenoux, 54280 Seichamps
}

\begin{abstract}
Résumé
Deux expériences ont été menées en serre sur de jeunes plants, respectivement de sapins (Abies alba, nordmanniana et numidica) et d'épicéa (Picea abies). Différentes durées et intensités de desséchement du sol ont été appliqućes. Cette année-là, la croissance aérienne ainsi que les différences de croissance et de biomasse foliaire entre traitements ont été faibles chez les sapins, importantes chez l'épicéa.

En fin d'année, les concentrations des différents éléments dans les aiguilles des sapins étaient d'autant plus faibles que la sécheresse avait été plus marquée.

Chez l'épicéa, les prélèvements foliaires effectués en cours de saison de végétation fournissent un résultat à peu près identique au précédent. Par contre, en fin d'année cela n'est plus valable que pour le potassium; au contraire, le déficit hydrique a alors augmenté les teneurs foliaires en azote, manganèse et calcium.

Il en découle, et pour cet élément cela concorde avec les renseignements bibliographiques, que l'analyse du potassium apparaît comme un test robuste de diagnostic de déficit hydrique sur de jeunes résineux, qui reste valable même après disparition du stress.

Parallèlement, une fertilisation en potassium pourrait peut-être corriger en partie les conséquences de la sécheresse.
\end{abstract}

\section{Introduction}

Un déficit dans l'alimentation hydrique des végétaux affecte leur nutrition minérale. L'eau jouc en effet un rôle important dans le transport des éléments nutritifs jusquaux racines (VIETs, 1972); en outre, le desséchement du sol réduit la biomasse racinaire formée au cours de l'année, aussi bien pour les plantes cultivées annuelles (GRINGRICH \& Russel, 1957) que pour les espèces ligneuses, et en particulier les jeunes plants (ZAHNER, 1968 ; BECKER, 1977). 
Il est également bien connu qu'un déficit hydrique inhibe aussi la croissance aérienne des plantes; cette réduction d'allongement de la tige s'accompagne, en particulier chez les espèces ligneuses, d'une diminution de la biomasse foliaire formée (Kozlowski, 1982).

Il résulte de ces deux phénomènes que la concentration (ou teneur, exprimée en p. 100) d'un élément dans les feuilles peut diminuer ou augmenter par rapport au témoin à la suite d'un stress hydrique, selon que ce dernier affecte plus l'assimilation de cet élément que la biomasse foliaire du végétal, ou que l'inverse se produise.

Cette modification de la teneur foliaire en certains éléments peut-elle être utilisée comme test de déficit hydrique, en complément de l'étude de facteurs plus immédiatement liés à ce stress, mais qui fluctuent beaucoup dans le temps, comme le potentiel hydrique des feuilles ou la résistance stomatique? Il faudrait, pour qu'elle présente un intérêt pratique, que cette modification soit sensible, qu'elle se produise toujours dans le même sens, qu'elle se maintienne dans différentes conditions de croissance aérienne et, si possible, qu'elle soit encore décelable lorsque des conditions favorables d'alimentation en eau se sont rétablies.

L'objectif de l'étude dont nous rendons compte ci-après est d'observer dans quelle mesure ces exigences sont satisfaites pour un ou plusieurs éléments, en vue de faciliter le diagnostic de déficit hydrique dans de jeunes plantations forestières. Les renseignements fournis par la bibliographie ne sont en effet pas toujours concordants, et surtout ne concernent que rarement des espèces ligneuses.

Cette étude pourrait en outre permettre de détecter des déficits minéraux provoqués par la sécheresse, déficits qui amplifient peut-être linfluence défavorable propre du manque d'eau. Dans ce cas un apport d'engrais pourrait s'avérer utile pour corriger en partie les conséquences de la sécheresse.

Nous nous sommes intéressés à de jeunes résineux, espèces très utilisées dans les reboisements. Afin de pouvoir contrôler le caractère assez général des résultats, nous avons considéré :

- 2 genres différents (épicéa commun et trois espèces de sapin),

- différentes intensités et périodes d'application de la sécheresse,

- un cas où la sécheresse provoque de fortes différences de croissance aérienne et un autre où ces différences sont très faibles (la croissance du témoin étant elle-même peu importante).

Nous nous limiterons strictement aux objectifs énoncés ci-dessus. Nous n'étudierons en particulier pas l'évolution des teneurs foliaires avec le temps, ce qui amènerait à émettre des hypothèses que nous ne sommes pas en mesure de vérifier.

\section{Matériel et méthodes}

\subsection{L'expérience sur les sapins}

Au cours d'une expérience rapportée par ailleurs (BECKER, 1977), ont été étudiées la transpiration et l'adaptation à la sécheresse de trois espèces de sapins : le sapin pectiné (Albies alba Mill.), le sapin de Nordmann (A. nordmanniana Spach.) et le sapin de Numidie ( $A$. numidica de Lannoy). 
L'expérience, menée en pots de culture, porte sur 25 semis d'un an de chacune des trois espèces, et se déroule sur deux saisons complètes de végétation. Le substrat utilisé est un mélange comprenant : 2 parties (en poids) de sable grossier, 3 de sable fin et 2 d'un mull mésotrophe à texture limono-argileuse. Au cours de la première année, les plants sont répartis en trois lots soumis à des modalités d'alimentation en eau très contrastées :

- modalité 1 : aucune restriction (témoin);

- modalité 2 : alimentation conçue de façon à provoquer une sécheresse marquée au cours de l'été ( $\mathrm{pF}$ du sol : 3,3 le 10 juillet; 4,2 le $1^{\text {er }}$ septembre);

- modalité 3 : alimentation conçue de façon à provoquer la sécheresse dès le printemps ( $\mathrm{pF} 3,3$ le 20 mai ; 4,2 le 20 juillet).

Une partie des plants sont sacrifiés à la fin de la première année. Les autres sont conservés en culture une année de plus, sans restriction d'alimentation en cau, afin d'observer les effets différés de la sécheresse de première année.

Parmi les résultats obtenus alors, on observe que la sécheresse inhibe fortement la croissance racinaire dès la première année et, à un degré moindre, la croissance en diamètre ; mais la croissance en longueur n'est pratiquement pas touchée ${ }^{(1)}$. Les autres effets ne se manifestent qu'au cours de la deuxième saison : retard au débourrement, diminution du nombre d'aiguilles et crosissance en longueur très amoindrie; par contre, croissance en diamètre et croissance racinaire retrouvent un niveau normal. Pour l'exposé complet des résultats, on se reportera à l'article cité (BECKER, 1977).

L'analyse minérale est effectuée sur différents types d'aiguilles :

- celles de l'année $n$ récoltées à la fin de l'année $n$ (appelées « aiguilles $n / n$ »),

- celles de l'année $n$ récoltées à la fin de l'année $\mathrm{n}+1$ (appelées «aiguilles $\mathrm{n} / \mathrm{n}+1 \gg)$,

- celles de l'année $\mathrm{n}+1$ récoltées à la fin de l'année $\mathrm{n}+1$ (appelées «aiguilles $\mathrm{n}+1 / \mathrm{n}+1 \gg)$.

Pour chaque espèce et chaque modalité, l'échantillon analysé est constitué par la totalité des aiguilles (de la catégorie indiquée) de trois plants différents.

\subsection{L'expérience sur l'épicéa commun (Picea abies (L.) Karsten)}

Elle est réalisée en serre dans des vases de végétation cylindriques d'environ $30 \mathrm{~cm}$ de hauteur et diamètre, contenant du sable surmonté, sur $7 \mathrm{~cm}$ de haut, d'un mull mésotrophe à texture limono-argileuse. Des semis de 2 ans sont repiqués, à raison de 9 par vase, au début de l'année de l'expérience. Des sondes hygrométriques de plâtre, installées dans le mull, permettent d'apprécier très grossièrement le $\mathrm{pF}$ du sol. sives :

L’expérience comporte 4 répétitions au départ, et se déroule en 2 phases succes-

(1) L'allongement axial est faible dans tous les cas (moyennes calculées pour les trois espèces : $33,0 \mathrm{~mm}$ en modalité $1,32,3 \mathrm{~mm}$ en modalité 2 et $27,3 \mathrm{~mm}$ en modalité 3 ), ce qui est normal pour des semis de sapin de 1 an. De plus cet allongement est acquis très tôt au printemps, avant que ne sc fasse sentir la sécheresse de modalité 2 et alors que celle de modalité 3 est encore peu marquée; ceci doit expliquer en grande partie la quasi-absence de répercussion des traitements sur les allongements. 
a) Jusqu'à la fin juillet, il y a 3 modalités :

- le témoin $\mathrm{C}$ : le sol est maintenu, grâce à des apports d'eau très fréquents, à un $\mathrm{pF}$ voisin de 2,5 , ce qui correspond à la capacité au champ ;

- la sécheresse $S 1$ : on laisse le sol se dessécher jusqu'à un pF de 3,3, puis on arrose un peu de façon à ce que le $\mathrm{pF}$ redescende de quelques dizièmes, on laisse à nouveau le sol se dessécher, etc.;

- la sécheresse S2 : même principe, mais en laissant le pF monter à 3,9.

Le desséchement du sol débute le 12 mai, alors que la pousse terminale vient de démarrer. Jusqu'à la fin juillet, on est amené à effectuer des apports d'eau à 8 reprises en $\mathrm{S} 1$, et à 4 reprises en $\mathrm{S} 2$. Les $\mathrm{pF}$ de $\mathrm{S} 1$ et $\mathrm{S} 2$ sont à peu près équivalents jusqu'au 5 juin.

b) A la fin du mois de juillet, après réhumectation de tous les seaux, 2 traitements sont appliqués :

- C : capacité au champ,

- $\mathrm{S}$ : desséchement du sol jusqu'à un pF de 3,9 puis réhumectation, etc.

Les traitements $\mathrm{C}$ et $\mathrm{S} 1$ de la première phase sont ainsi scindés en deux; les modalités sont donc les suivantes au cours de la deuxième phase : $\mathrm{CC}, \mathrm{CS}, \mathrm{S} 1 \mathrm{C}, \mathrm{S} 1 \mathrm{~S}$, S2C.

On observe de fortes différences de croissance en longueur et de biomasse foliaire entre les divers traitements.

Les prélèvements foliaires destinés aux analyses ont été effectués les 20-6, 27-7 et 15-12. Chaque échantillon comporte un mélange de l'ensemble des aiguilles de l'année de 2 ou 3 plants. Il n'y a pas de répétition à chaque date, en raison de la faible biomasse foliaire formée dans certains traitements.

\section{Résultats}

\subsection{L'expérience sur les sapins}

Le tableau 1 indique, pour mémoire, les valeurs obtenues précédemment pour la transpiration cumulée des plants. Celles-ci donnent une idée de l'intensité des sécheresses imposées au cours de lannée $n$, en particulier en modalité 3 (sécheresse dès le printemps), pour laquelle la transpiration est 3 à 4 fois plus faible que celle de modalité 1 (pas de restriction).

Les différences observées pour l'année $n+1$ doivent être examinées en prenant en compte les réductions de taille des plants consécutives aux sécheresses subies au cours de l'année $n$.

Les résultats concernant la nutrition sont présentés sur la figure 1 et le tableau 2. 


\section{TABleau 1}

Transpiration cumulée par plant en fonction des modalités d'alimentation en eau imposées au cours de lannée $n$.

Cumulated transpiration per plant related with the treatments of water supply during year $n$.

\begin{tabular}{|c|c|c|c|}
\hline & & \multicolumn{2}{|c|}{ Transpiration cumulée (g) } \\
\hline & & année $\mathrm{n}$ & année $\mathbf{n}+\mathbf{1}$ \\
\hline A. alba & 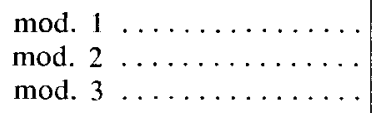 & $\begin{array}{r}1803 \\
1202 \\
447\end{array}$ & $\begin{array}{ll}3 & 683 \\
3 & 079 \\
2 & 885\end{array}$ \\
\hline A. nordmanniana & 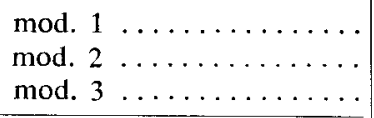 & $\begin{array}{r}1671 \\
913 \\
389 \\
\end{array}$ & $\begin{array}{ll}3 & 197 \\
1 & 898 \\
2 & 221 \\
\end{array}$ \\
\hline A. numidica & 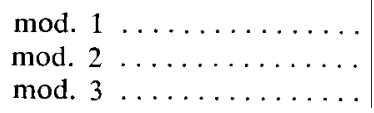 & $\begin{array}{r}1377 \\
944 \\
474\end{array}$ & $\begin{array}{l}3853 \\
2674 \\
2464\end{array}$ \\
\hline
\end{tabular}

\section{TABLEAU 2}

Teneurs foliaires en éléments minéraux (expérience sur les sapins). Foliar contents in mineral elements (experiment on the firs).

$\mathrm{n} / \mathrm{n}$ (aiguilles formées l'année $n$ et analysées l'année $n$ )

\begin{tabular}{l|c|c|c|c|c|c|c} 
& $\mathrm{N}(\%)$ & $\mathrm{P}(\%)$ & $\mathrm{K}(\%)$ & $\mathrm{Ca}(\%)$ & $\mathrm{Mg}(\%)$ & $\mathrm{Mn}(\%)$ & $\mathrm{Si}(\%)$ \\
\hline Mod. $1 \ldots \ldots \ldots$ & 1,90 & 0,22 & 0,58 & 0,87 & 0,18 & 0,28 & 2,01 \\
Mod. 2 $\ldots \ldots \ldots$ & 1,45 & 0,13 & 0,51 & 0,53 & 0,14 & 0,14 & $\mathbf{1 , 6 2}$ \\
Mod. 3 $\ldots \ldots \ldots$ & 1,37 & 0,10 & 0,36 & 0,50 & 0,11 & 0,13 & 2,20
\end{tabular}

$\mathrm{n} / \mathrm{n}+1$ (aiguilles formées l'année $n$ et analysées l'année $\boldsymbol{n}+1$ )

\begin{tabular}{l|c|c|c|c|c|c|c} 
& $\mathrm{N}(\%)$ & $\mathrm{P}(\%)$ & $\mathrm{K}(\%)$ & $\mathrm{Ca}(\%)$ & $\mathrm{Mg}(\%)$ & $\mathrm{Mn}(\%)$ & $\mathrm{Si}(\%)$ \\
\hline Mod. $1 \ldots \ldots \ldots$ & 1,02 & 0,12 & 0,64 & 1,25 & 0,24 & 0,46 & $\mathbf{1 , 7 4}$ \\
Mod. 2 $\ldots \ldots \ldots$ & 1,27 & 0,13 & 0,50 & 1,07 & 0,24 & 0,38 & 2,00 \\
Mod. 3 $\ldots \ldots \ldots$ & 1,16 & 0,13 & 0,52 & 1,05 & 0,20 & 0,34 & $\mathbf{1 , 9 3}$
\end{tabular}

$\mathrm{n}+1 / \mathrm{n}+1$ (aiguilles formées l'année $n+1$ et analysées l'année $n+1$ )

\begin{tabular}{l|c|c|c|c|c|c|c} 
& $\mathrm{N}(\%)$ & $\mathrm{P}(\%)$ & $\mathrm{K}(\%)$ & $\mathrm{Ca}(\%)$ & $\mathrm{Mg}(\%)$ & $\mathrm{Mn}(\%)$ & $\mathrm{Si}(\%)$ \\
\hline Mod. $1 \ldots \ldots \ldots$ & $\mathbf{0 , 7 8}$ & $\mathbf{0 , 1 6}$ & 0,61 & 0,72 & 0,20 & $\mathbf{0 , 3 7}$ & $\mathbf{1 , 4 3}$ \\
Mod. 2 $\ldots \ldots \ldots$ & $\mathbf{1 , 1 8}$ & 0,16 & 0,57 & $\mathbf{0 , 8 2}$ & 0,22 & 0,35 & $\mathbf{1 , 7 1}$ \\
Mod. 3 $\ldots \ldots \ldots$ & $\mathbf{1 , 1 1}$ & 0,15 & 0,56 & 0,82 & 0,19 & $\mathbf{0 , 3 2}$ & $\mathbf{1 , 4 1}$ \\
\hline
\end{tabular}

Mod. 1 : témoin sans restriction d'eau.

Mod. 2 : sécheresse tardive (année $n$ seulement).

Mod. 3 : sécheresse précoce et prolongée (année n seulement).

Treatment 1 : control with good water supply.

Treatment 2 : later soil drying (year $n$ only).

Treatment 3 : earlier and longer soil drying (year $n$ only). 

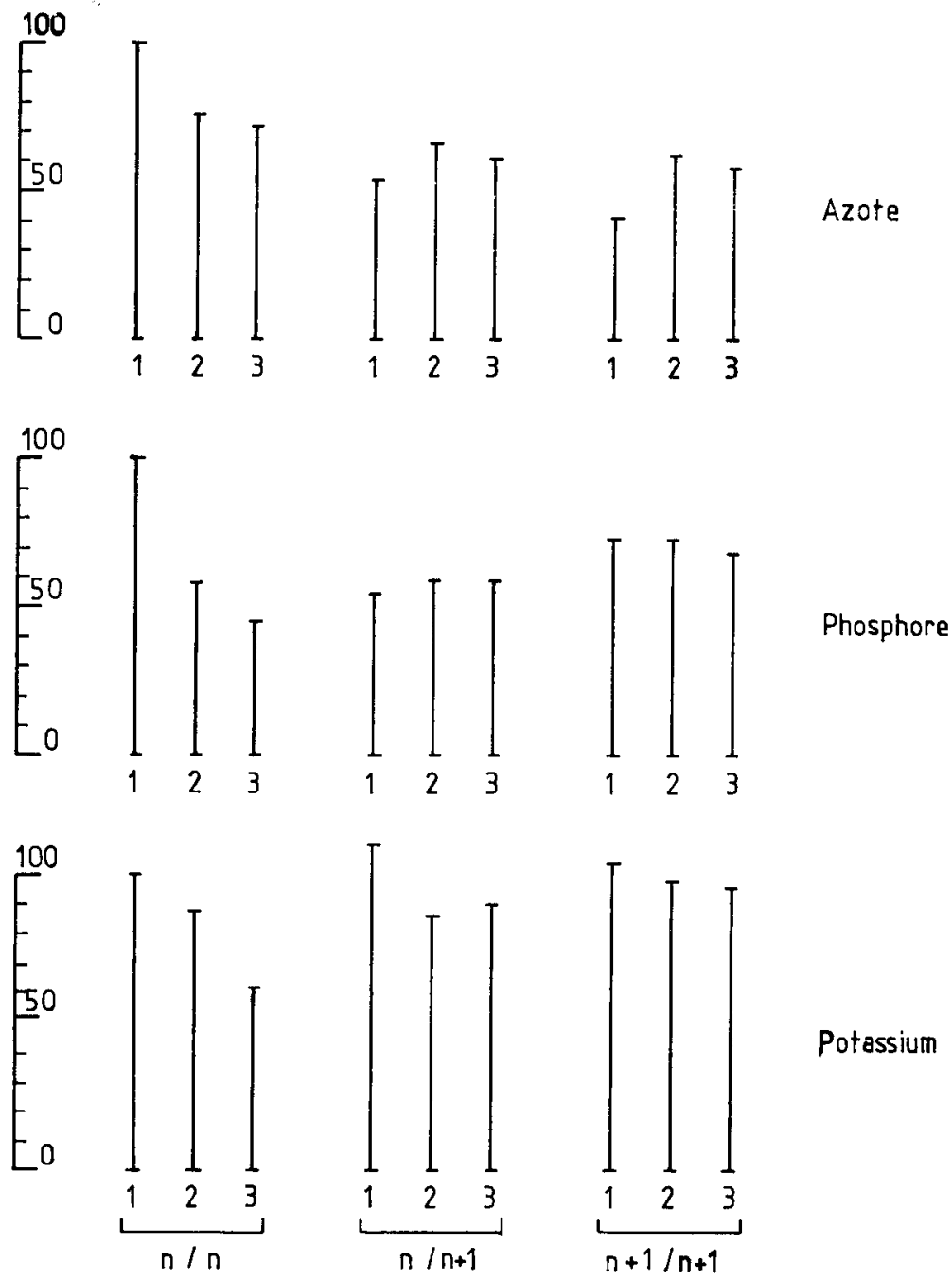

FIG. 1

Teneurs relatives en $N, P$ et $K$, en ramenant à 100

la teneur des aiguilles $n / n$ de modalité 1 (expérience sur les sapins).

Relative contents in $N, P$ and $K$;

the content of needles $n / n$ for treatment 1 been brought up to 100 (experiment on the firs).

1 : témoin bien alimenté en eau.

2: sécheresse tardive.

3 : sécheresse précoce et prolongée.

$\mathrm{n} / \mathbf{n}$ : aiguilles formées l'année $\mathrm{n}$ et analysées l'année $\mathrm{n}$.

$\mathrm{n} / \mathrm{n}+1$ : aiguilles formées l'année $\mathrm{n}$ et analysées l'année $(\mathrm{n}+1)$.

$\mathbf{n}+1 / \mathbf{n}+\mathbf{1}$ : aiguilles formées l'année $(\mathbf{n}+1)$ et analysées l'année $(n+1)$. 
Nous n'avons pas décelé de différences de comportement significatives entre les 3 espèces étudiées; aussi l'interprétation des résultats porte-t-elle sur les moyennes de ces 3 espèces.

\subsection{Aiguilles $n / n$}

Pour tous les éléments, sauf le silicium, les teneurs foliaires se classent dans l'ordre suivant : modalité $1>$ modalité $2>$ modalité 3 , les différences entre 2 modalités successives étant parfois très nettes, parfois beaucoup moins. Elles sont nettes pour tous les éléments entre les modalités 1 et 2 ; elles sont très faibles entre les modalités 2 et 3 pour le calcium et le manganèse.

La pousse aérienne du sapin étant peu affectée par la sécheresse au cours de cette première année, ces résultats traduisent l'influence réelle de la sécheresse sur la capacité d'absorption des différents éléments par le système racinaire.

\subsection{Aiguilles $n+1 / n+1$}

Les teneurs foliaires en éléments sont en général peu liées aux conditions d'alimentation en eau au cours de l'année précédente, en tout cas nettement moins que pour les aiguilles $\mathrm{n} / \mathrm{n}$. Dans les plants soumis précédemment à un stress, les teneurs relatives ont fortement remonté par rapport au témoin, ce qui est logique, car leur développement racinaire au cours de l'année $n+1$ est normal, alors que leur croissance aérienne en longueur est fortement réduite.

\subsection{Aiguilles $n / n+1$}

Leurs teneurs foliaires ne sont non plus pas très liées aux conditions d'alimentation en eau au cours de l'année $n$, nettement moins de toute façon que les aiguilles $n / n$.

\subsection{L'expérience sur l'épicéa}

Le tableau 3 présente les teneurs des différents éléments dans les feuilles, par traitement, pour chacune des 3 dates de prélèvement.

Pour tous les éléments analysés, sauf le silicium, nous observons, lors de la deuxième date d'analyse (27-7), le classement suivant : $\mathrm{C}>\mathrm{S} 1>\mathrm{S} 2$.

Lors de la première date (20-6), l'ordre des traitements était déjà le même pour le potassium, le calcium, le magnésium et le manganèse. L'azote et le phosphore faisaient plus ou moins exception à la règle ; remarquons qu'à cette date, $\mathrm{S} 2$ ne correspondait pas encore à un desséchement du sol nettement plus prononcé que S1.

Lors de la troisième date (15-12), certains éléments montrent toujours un même sens de variation dans toutes les comparaisons que l'on peut effectuer, que la différence d'alimentation en eau soit encore appliquée lors du prélèvement (comparaisons : $\mathrm{CC}$ et S1S ; S1C et S1S ; CC et CS) ou qu'elle ait cessé depuis fin juillet (comparaisons : $\mathrm{CC}, \mathrm{S} 1 \mathrm{C}$ et $\mathrm{S} 2 \mathrm{C} ; \mathrm{CS}$ et $\mathrm{S} 1 \mathrm{~S})$ :

- la sécheresse diminue ainsi la teneur en $\mathbf{K}$, et d'autant plus qu'elle est plus prononcée ou plus longue ( $\mathrm{P}$ a tendance à se comporter de la même manière, mais les différences de concentration en cet élément sont presque toujours très peu marquées) ; - le résultat est inverse pour $\mathrm{N}$ et $\mathrm{Mn}$ (et presque toujours pour $\mathrm{Ca}$ ). 
TABleaU 3

Teneurs foliaires en éléments minéraux (expérience sur épicéa).

Foliar contents in mineral elements (experiment on the spruce).

\begin{tabular}{|c|c|c|c|c|c|c|c|c|c|}
\hline Date & $\begin{array}{c}1^{\text {ro }} \\
\text { phase }\end{array}$ & $2^{\mathrm{e}}$ phase & $N(\%)$ & $\mathrm{P}(\%)$ & $\mathrm{K}(\%)$ & $\mathrm{Ca}(\%)$ & $\operatorname{Mg}(\%)$ & $\operatorname{Mn}(\%)$ & Si $(\%)$ \\
\hline $20-6$ & $\begin{array}{l}\text { C } \\
\text { S1 } \\
\text { S2 }\end{array}$ & & $\begin{array}{l}1,54 \\
1,65 \\
1,31\end{array}$ & $\begin{array}{l}0,11 \\
0,10 \\
0,10\end{array}$ & $\begin{array}{l}0,96 \\
0,84 \\
0,80\end{array}$ & $\begin{array}{l}0,68 \\
0,62 \\
0,50\end{array}$ & $\begin{array}{l}0,12 \\
0,11 \\
0,09\end{array}$ & $\begin{array}{l}0,64 \\
0,50 \\
0,34\end{array}$ & $\begin{array}{l}0,61 \\
0,62 \\
0,46\end{array}$ \\
\hline $27-7$ & $\begin{array}{l}C \\
\text { S1 } \\
\text { S2 }\end{array}$ & & $\begin{array}{l}2,30 \\
2,07 \\
1,78\end{array}$ & $\begin{array}{l}0,17 \\
0,12 \\
0,10\end{array}$ & $\begin{array}{l}0,88 \\
0,80 \\
0,74\end{array}$ & $\begin{array}{l}1,06 \\
0,86 \\
0,84\end{array}$ & $\begin{array}{l}0,14 \\
0,12 \\
0,09\end{array}$ & $\begin{array}{l}1,61 \\
1,24 \\
1,21\end{array}$ & $\begin{array}{l}0,82 \\
0,76 \\
0,87\end{array}$ \\
\hline $15-12$ & $\begin{array}{l}\text { C } \\
\text { S1 } \\
\text { S2 }\end{array}$ & $\begin{array}{l}\mathrm{C} \\
\mathrm{C} \\
\mathrm{C}\end{array}$ & $\begin{array}{l}1,66 \\
2,00 \\
2,19\end{array}$ & $\begin{array}{l}0,26 \\
0,25 \\
0,25\end{array}$ & $\begin{array}{l}0,90 \\
0,74 \\
0,52\end{array}$ & $\begin{array}{l}1,45 \\
1,35 \\
1,79\end{array}$ & $\begin{array}{l}0,13 \\
0,11 \\
0,15\end{array}$ & $\begin{array}{l}2,20 \\
2,40 \\
4,00\end{array}$ & $\begin{array}{l}2,30 \\
1,30 \\
2,43\end{array}$ \\
\hline $15-12$ & $\begin{array}{l}\mathrm{C} \\
\mathrm{S} 1\end{array}$ & $\begin{array}{l}S \\
S\end{array}$ & $\begin{array}{l}1,83 \\
2,07\end{array}$ & $\begin{array}{l}0,25 \\
0,24\end{array}$ & $\begin{array}{l}0,82 \\
0,38\end{array}$ & $\begin{array}{l}1,42 \\
2,14\end{array}$ & $\begin{array}{l}0,21 \\
0,17\end{array}$ & $\begin{array}{l}2,40 \\
3,60\end{array}$ & $\begin{array}{l}0,99 \\
1,47\end{array}$ \\
\hline
\end{tabular}

Synthétiquement, si l'on compare les résultats des analyses correspondant aux trois dates de prélèvement, on constate que, même si le prélèvement est effectué bien après cessation du stress : en $K$;

- une sécheresse se traduit toujours par une diminution de la teneur foliaire

- pour $\mathrm{N}$ et surtout $\mathrm{Mn}$, le résultat est identique à celui de $\mathrm{K}$ si le prélèvement est relativement précoce, il est inverse si le prélèvement est très tardif.

\section{Discussion et conclusion}

Nos résultats concordent-ils avec les renseignements fournis par la bibliographie? Les auteurs ayant étudié ce problème, principalement sur des plantes de cultures (par exemple Hernando et al., 1968 ; Viets, 1972 ; Richards \& Wadleigh, 1952 ; Mederski \& Wilson, 1960 ; Kramer \& Kozlowski, 1979) font souvent état, comme conséquence de la sécheresse, d'une augmentation de la teneur foliaire en azote et d'une diminution de la concentration en phosphore et/ou potassium, parfois de symptômes de carence en manganèse ou en fer, et en général de teneurs variables en calcium et en magnésium. Dans le domaine des espèces forestières résineuses, LAATSCH (1976) signale des déficiences en potassium entraînées par la sécheresse sur pin d'Alep en climat méditerranéen, sur sols calcaires superficiels.

S'il y a concordance avec nos résultats pour le potassium, on ne peut affirmer que ce soit vraiment le cas pour le phosphore, les différences entre traitements étant 
très peu marquées pour cet élément dans l'expérience sur épicéa. Quant à l'azote, si nous avons effectivement une teneur plus élevée pour les traitements «secs » que pour les témoins en fin d'année sur épicéa, le contraire s'était produit lors des prélèvements antérieurs, ainsi que sur les sapins en fin d'année; comme nous l'avons indiqué précédemment, le sens dans lequel la sécheresse fait évoluer la teneur d'un élément dans les feuilles dépend de la variable qui est la plus affectée : assimilation de l'élément considéré ou biomasse foliaire formée. Le tableau 4 illustre bien ce propos dans le cas de l'azote : la quantité d'azote assimilée par l'épicéa est très affectée par la sécheresse (et d'autant plus que celle-ci a été plus longue ou plus sévère), beaucoup plus que dans le cas des sapins, mais moins que la biomasse foliaire; c'est le contraire pour les sapins.

\section{Tableau 4}

Biomasse foliaire relative et azote en fin de $1^{\text {re }}$ année.

Relative foliar biomass, and nitrogen, at the end of the first year.

\begin{tabular}{|c|c|c|c|c|c|c|c|c|}
\hline \multirow{2}{*}{ Modalités } & \multicolumn{3}{|c|}{ Sapins } & \multicolumn{5}{|c|}{ Epicéa } \\
\hline & 1 & 2 & 3 & $\mathrm{CC}$ & $\mathrm{CS}$ & $\mathrm{S} 1 \mathrm{C}$ & $\mathrm{S} 2 \mathrm{C}$ & S1S \\
\hline $\begin{array}{c}\text { B:omasse foliaire relative } \\
\text { moyerne (témoin ramené } \\
\left.\text { à } 100^{\circ}\right) \ldots \ldots \ldots \ldots \ldots\end{array}$ & 100 & 94,4 & 91,8 & 100 & 85,9 & 60,3 & 26,6 & 22,0 \\
\hline$N(\%) \ldots \ldots \ldots \ldots$ & 1,90 & 1,45 & 1,37 & 1,66 & 1,83 & 2,00 & 2,19 & 2,07 \\
\hline $\begin{array}{l}\mathbf{N} \text { total relatif dans l'en- } \\
\text { semble de la biomasse } \\
\text { foliaire } \ldots \ldots \ldots \ldots \ldots \ldots\end{array}$ & 190,0 & 136,9 & 125,8 & 166,0 & 157,2 & 120,6 & 58,3 & 45,5 \\
\hline
\end{tabular}

On s'explique ainsi que la teneur en azote des feuilles, en p. 100 de la matière sèche, ait augmenté dans le cas de l'épicéa et diminué dans le cas des sapins.

Si nous revenons à l'objectif de cette étude tel que nous l'avons énoncé dans l'introduction, notre attention se porte fortement, et presque uniquement, sur le potassium. Pour les deux séries d'espèces utilisées dans nos expériences, dans des conditions dissemblables de croissance aérienne, la sécheresse a toujours provoqué une diminution de la teneur foliaire en cet élément par rapport au témoin, diminution d'autant plus importante que le stress avait été plus sévère ou d'une durée plus longue ; ce phénomène se maintient après rétablissement de conditions favorables d'alimentation en eau (par contre, il disparaît au cours de l'année suivant le stress). L'analyse du potassium apparaît ainsi comme un test robuste de diagnostic de déficit hydrique sur de jeunes résineux, sans doute parce que l'assimilation de cet élément est très nettement plus affectée que la formation de biomasse nouvelle.

On peut espérer que cette conclusion reste valable sur d'autres espèces que celles utilisées, ainsi que sur des sujets installés depuis plus longtemps que dans le cas de nos 
expériences; mais cela reste à vérifier (elle ne s'applique d'une manière rigoureuse, par analogie avec nos expériences, qu'à des plantations de $1^{\text {re }}$ année, dont le système racinaire n'est donc pas encore bien établi). En outre, du moins en théorie, il n'est pas impossible que la tendance s'estompe, ou même s'inverse, dans certains cas où la limitation de la biomasse foliaire par le déficit hydrique serait encore plus marquée. Enfin, les teneurs foliaires en potassium dépendent en partie de la richesse du sol en cet élément; on comparera donc de préférence ces teneurs dans des plantations situées sur un même substrat : par exemple, comparaison soit à deux années différentes dans une même station, soit à un même moment, mais dans des situations topographiques variées ; si l'on examine au contraire les résultats d'une analyse isolée et que la teneur en potassium apparaisse faible, il faudra vérifier que le sol n'est pas spécialement pauvre en cet élément avant de conclure à un déficit hydrique.

Notre étude a ainsi permis de vérifier que les données bibliographiques concernant le potassium étaient applicables à de jeunes résineux, et que l'amplitude d'utilisation du test était très large ; par contre, les renseignements recueillis dans la littérature sur les autres éléments nutritifs ne semblent pas, et de loin, avoir une portée aussi générale.

En dehors des éléments nutritifs proprement dits, certains auteurs (par exemple Bartoli \& Souchier, 1978) ont montré que la teneur foliaire en silicium augmentait avec la transpiration de la plante, ce qui pourrait laisser entendre qu'elle serait un bon indicateur de son alimentation en eau. Nos résultats ne confirment pas cet espoir.

Reçu pour publication en décembre 1982.

\section{Summary \\ Soil drying and mineral nutrition of young plants of coniferous species.}

Two experiments habe been realized in a greenhouse on young plants of firs (Abies alba, nordmanniana, numidica) and spruce (Picea abies). Different durations and intensities of soil drying have been carried out. That year, the shoot increase as well as the differences of growth and new leaf area between treatments were small for firs and important for spruce.

At the end of the year, the more important had been the soil desiccation, the smaller were the different elements contents in the firs needles.

On spruce, the results are about the same with sampling during the growth period. On the other hand, at the end of the year, it is not the case any longer, except for potassium; on the contrary, the water deficit had then increased the needles contents in nttrogen, manganese and calcium.

Analysis of foliar potassium content appears therefore (ours results agree with litterature for this element) as a good test for water deficit diagnosis on young plants of conifers; this test remains available even after the stress has disappeared.

Concurrently, soil fertilization with potassium could perhaps reduce the effects of soil dessication on these plants. 


\section{Références bibliographiques}

Bartoli F., Souchier B., 1978. Cycle et rôle du silicium d'origine végétale dans les écosystèmes forestiers tempérés. Ann. Sci. for., 35 (3), 187-202.

Baule F., Fricker C., 1969. La fertilisation des arbres forestiers. BLV, München, $256 \mathrm{p}$.

BeCKer M., 1977. Contribution à l'étude de la transpiration et de l'adaptation à la sécheresse des jeunes plants résineux. Exemple de trois sapins du pourtour méditerranéen (Abies alba, A. nordmanniana, A. numidica). Ann. Sci. for., 34 (2), 137-158.

Bradbury I.K., Malcolm D.C., 1977. The effect of phosphorus and potassium on transpiration, leaf diffusive resistance and water-use efficiency in Sitka Spruce (Picea sitchensis) seedlings. J. appl. Ecol., 14 (2), 631-641.

GringRich J.R., RUSSEL M.B., 1957. A comparison of effects of soil moisture tension and osmotic stress on root growth. Soil Sci., 84, 185-194.

Hernando V. et al., 1968. Effect of soil moisture on the mineral nutrition of the plant. Agrochimica, 12, 341-352.

Kozlowski T.T., 1982. Water supply and tree growth. Part I Water deficits. For. Abstr., $43(2), 57-95$.

Kramer P.J., Kozlowski T.T., 1979. Physiology of woody plants. Academic Press, New York, 811 p.

LaATsCH W., 1967. Beziehungen zwischen Standort, Ernährungszustand und Wuchsleistung von Kiefernaufforstungen im Mittelmeergebiet. Forstw. Centr., 86 (2), 69-81.

Mederski H.J., WILSON J.H., 1969. Relation of soil moisture to ion absorption by corn plants. Soil Sci. Soc. Am. Proc., 24, 149-152.

Richards L.A., Wadleigh, 1952. Soil water and plant growth. In "Soil physical conditions and plant growth»(B.T. Shaw ed.), Academic Press, New York, 73-251.

VIETS F.G., 1972. Water deficits and nutrient availability, 217-239. In "Water deficits and plant growth», vol. III "Plant responses and control of water balance» (Т.T. Kozlowski ed.), Academic Press, New York, 368 p.

ZAHNER R., 1968. Water deficits and growth of trees, 191-254. In «Water deficits and plant growth», vol. II "Plant water consumption and response» (T.T. Kozlowski ed.), Academic Press, New York, 333 p. 